\title{
Modelling Oil Price with Lie Algebras and Long Short-Term Memory Networks
}

\author{
Melike Bildirici ${ }^{1, *}$, Nilgun Guler Bayazit ${ }^{2}$ and Yasemen Ucan ${ }^{2}$ \\ 1 Department of Economics, Yildiz Technical University, 34220 Istanbul, Turkey \\ 2 Department of Mathematical Engineering, Yildiz Technical University, 34220 Istanbul, Turkey; \\ guler@yildiz.edu.tr (N.G.B.); ucan@yildiz.edu.tr (Y.U.) \\ * Correspondence: bildiri@yildiz.edu.tr
}

Citation: Bildirici, M.; Bayazit, N.G.; Ucan, Y. Modelling Oil Price with Lie Algebras and Long Short-Term Memory Networks. Mathematics 2021, 9, 1708. https://doi.org/10.3390/ math9141708

Academic Editor: Theodore E. Simos

Received: 2 May 2021

Accepted: 11 July 2021

Published: 20 July 2021

Publisher's Note: MDPI stays neutral with regard to jurisdictional claims in published maps and institutional affiliations.

Copyright: (C) 2021 by the authors. Licensee MDPI, Basel, Switzerland. This article is an open access article distributed under the terms and conditions of the Creative Commons Attribution (CC BY) license (https:// creativecommons.org/licenses/by/ $4.0 /)$.

\begin{abstract}
In this paper, we propose hybrid models for modelling the daily oil price during the period from 2 January 1986 to 5 April 2021. The models on $S^{2}$ manifolds that we consider, including the reference ones, employ matrix representations rather than differential operator representations of Lie

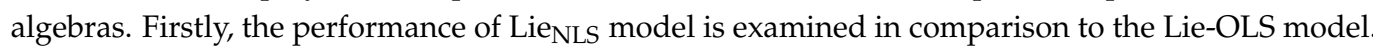
Then, both of these reference models are improved by integrating them with a recurrent neural network model used in deep learning. Thirdly, the forecasting performance of these two proposed

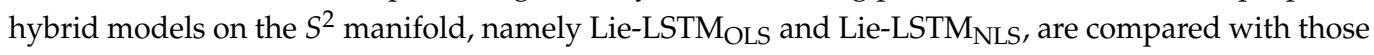
of the reference Lie OLS and Lie ${ }_{\text {NLS }}$ models. The in-sample and out-of-sample results show that our proposed methods can achieve improved performance over Lie $\mathrm{OLS}_{\mathrm{S}}$ and Lie $\mathrm{NLS}_{\mathrm{S}}$ models in terms of RMSE and MAE metrics and hence can be more reliably used to assess volatility of time-series data.
\end{abstract}

Keywords: oil price forecasting; Lie group SO(3); LSTM; deep learning; short-term model

\section{Introduction}

Crude oil is a strategic natural resource since it is a commodity connected with many financial instruments, such as futures, options, and bonds. While most financial instruments have a short-term maturity period, there are cases with long-term pricing maturity for oil. Additionally, crude oil price has nonlinear behavior.

The nonlinear behavior in the oil price has been well discussed and analyzed by many articles in the past. Barone-Adesi et al. [1] suggested a semiparametric method to examine the structure of oil prices. Adrangi et al. [2] determined the presence of low-dimensional chaotic structure in the oil prices. Lahmiri [3], Bildirici, and Sonustun [4]; Komijani et al. [5]; and $\mathrm{He}[6]$ are the other studies that determine the presence of chaos in the oil prices. Bildirici et al. [7] suggested a new hybrid modelling technique based on the LSTARGARCH and LSTM models to analyze the volatility of oil prices.

Apart from the works on volatility, the works by [8,9] carry importance. Gibson and Schwartz [8] also shows "the mean reverting tendency as well as the variability of its changes requires a stochastic representation in order to price oil-linked securities accurately". In [9], a model that depends on a two-factor model for pricing financial and real assets contingent on the price of oil is developed. For valuing futures contracts, the parameters of the model were estimated by using the data between January 1984 and November 1988, and the model was tested on the out of sample data between November 1988 and May 1989. The purpose of the current work is to offer an approach applicable to pricing based on the Lie method.

In this paper, we employ Lie algebras method to solve stochastic differential equation (DE) of short-term model of the oil. We suggest that the model is governed by a stochastic differential equation model on a curved state space and develop oil price models using matrix representations and differential operator on the $S^{2}$ manifold. In late 19th century, under a continuous group of symmetries, Lie discovered that special approaches to solve 
DEs were special states of a general integration process dependent upon the invariance of the DE. Nowadays, the applications of Lie groups have a deep impact on the branches of mathematics, mechanics, and robotics sciences.

Especially, in mathematical finance, a few papers employed the Lie method to provide awareness to the structure of related partial differential equations. The approach of employing general differentiable manifolds in interest rate models appears in [10-12]. Gazizov and Ibragimov [11] used the Lie method in the context of Black-Scholes-Merton equation. Lo and Hui [13] and Carr et al. [14] constructed a concrete example of a short-rate model on the circle $S^{1}$. Park et al. [15] tested the proposition that nonlinear and random behavior of interest rates is governed by a stochastic differential equation model on a curved state space. They developed short-term interest rate models on $S^{1}$ and $S^{2}$ manifolds using matrix representations instead of differential operator representations of Lie groups.

In this paper, we employed spot price (WTI crude daily oil price) during the period from 2 January 1986 to 5 April 2021. The selected period includes some important events that had impacts on the oil price, such as multiple economic crises (1981, 2001, and 2008), US military intervention in Iraq, COVID-19, etc. These factors lead to nonlinear behavior in the oil price between spot and futures contracts.

Therefore, modeling dynamic processes and solving stochastic differential equations (SDE) are important. As is widely recognized, the solutions of DEs yield a set of symmetries that corresponds to Lie groups. In this paper, we employ a model on $S^{2}$ manifolds that uses matrix representations instead of differential operator representations of Lie algebras. As accented by [15], the drift and noise volatility terms of the stochastic state equations are worked out to reflect various observed phenomena. We try to keep these terms simple and instead choose an underlying state space that is curved. Park et al. [15] and Goard [16] used the ordinary least square (OLS) estimation method for parameter estimation. We preferred the nonlinear least square (NLS) method for parameter estimation due to the nonlinear behavior in the specified period.

As our primary contribution, we propose the use of LSTM networks for forecasting in the domain obtained by the Lie method. Specifically, we suggest both the hybrid Lie-

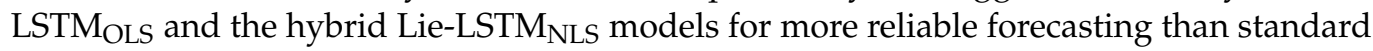
regression methods in this domain. The forecasting performances of our proposed hybrid methods, Lie-LSTM $\mathrm{OLS}_{\text {and Lie-LSTM }}$ NLS, are compared against those of the Lie NLS and Lie OLS standard regression methods on the WTI oil price data.

The paper is organized as follows. In Section 2, the orthogonal matrix Lie groups and algebras are given, and then the oil price model is defined on the Lie groups $\mathrm{SO}(3)$. In Section 3, the data is presented, and some of its descriptive statistics are given. In Section 4, results are presented and discussed, and the last section gives the conclusion.

\section{Materials and Methods}

\subsection{Preliminaries on Orthogonal Matrix Lie Groups and Algebras}

In this section, the definitions of orthogonal matrix Lie groups, their algebras, and the relations of stochastic dynamics with these groups are given [15,17].

As it is known, a geometrically Lie group is a differentiable manifold, and its algebra is the tangent space in the unit neighboring to the manifold. Usually, the group is denoted with a capital letter and algebra with a lowercase letter. Let $\mathrm{G}$ and $\mathrm{g}$ be a matrix Lie group with dimension $\mathrm{n}$ and its algebra, respectively. In this case, the orthogonal matrix groups are denoted as $O(n)$ and defined as follows:

$$
O(n)=\left\{A \in G L(n): A^{T} A=I\right\}
$$

Special orthogonal matrix groups are denoted as $S O(n)$ and defined as follows:

$$
S O(n)=\left\{A \in G L(n): A^{T} A=I \text { and } \operatorname{det} A=1\right\}
$$


The manifold of Lie group $\mathrm{SO}(2)$ is identified with the unit circle $S^{1}=\left\{\left(x_{1}, x_{2}\right): x_{1}^{2}+x_{2}^{2}=1\right\}$ with parametrization $x_{1}=\cos \theta, x_{2}=\sin \theta$.

The manifold of Lie group $\mathrm{SO}(3)$ is identified with the unit sphere $S^{2}=\left\{\left(x_{1}, x_{2}, x_{3}\right): x_{1}^{2}+x_{2}^{2}+x_{3}^{2}=1\right\}$, with parametrization $x_{1}=\cos \theta, x_{2}=\sin \theta \sin \varphi$, $x_{3}=\sin \theta \cos \varphi$. Similarly, the Lie group $S O(n)$ is identified with the $n-1$ dimensional manifolds $S^{n-1}$.

Lie algebras of these groups are denoted by $s o(n)$, and the elements of the algebra satisfy the condition $B^{T}=-B$ for $B \in s o(n)$. The relationship between this algebra and the group is expressed by:

$$
\begin{aligned}
& \exp : \operatorname{so}(3) \rightarrow S O(3) \\
& \quad \exp (B)=A \in S O(3)
\end{aligned}
$$

Proposition 1. ([15]). Bilinear state equation

$$
d A=A B d t+A d W
$$

where $A \in G$ and $B, d W \in g, A$ is a constant, and $d W$ is the diffusion process, and the quadratic function is $f(A)=\frac{1}{2} \operatorname{Tr}\left(A^{T} Q A N\right)$, where symmetric matrix $Q, N \in R^{n * n}$ are given. Hence the dynamics for $f$ are as follows:

$$
d f=\operatorname{Tr}\left[A^{T} Q A\left(B N d t+d W N+\frac{1}{2} d W N d W^{T}+\frac{1}{2} d W d W N\right)\right]
$$

where $d t . d t=d w_{i} d t=d t d w_{i}=0, d w_{i} d w_{j}=\rho_{i j} d t$, and $\rho_{i j}$ is the correlation coefficient between $w_{i}$ and $w_{j}$.

Proposition 2. ([15]). Under the conditions given in proposition 1, if $f(A)=\operatorname{Tr}(M A)$ and $M$ is symmetric, the dynamics for $f$ is given as

$$
d f=\operatorname{Tr}\left[M A\left(B d t+d W+\frac{1}{2} d W d W\right)\right]
$$

\subsection{Stochastic Dynamics on Orthogonal Matrix Lie Groups}

\subsubsection{The Lie Group SO(2)}

As it is known, the Lie group $S O(2)$ is a differentiable manifold, and this manifold can be identified with the unit circle $S^{1}$. The oil price is defined as follows: $s(A)=\operatorname{Tr}(M A)$, where $A \in S O(2)$ and $M$ is a symmetric positive definite matrix.

Thus the bilinear state equation is given:

$$
d A=A B d t+A d W \text { where } B, d W \in \text { so(2) }
$$

Indeed, for

$$
\begin{gathered}
M=\left[\begin{array}{cc}
m_{11} & m_{12} \\
m_{21} & m_{22}
\end{array}\right]>0 \text { and } A=\left[\begin{array}{cc}
\cos \theta & -\sin \theta \\
\sin \theta & \cos \theta
\end{array}\right], \theta \in[o, 2 \pi] \\
B=\left[\begin{array}{cc}
0 & -b \\
b & 0
\end{array}\right], d W=\left[\begin{array}{cc}
0 & -d w \\
d w & 0
\end{array}\right] \in \operatorname{so}(2) \\
s(A)=\left(m_{11}+m_{22}\right) \cos \theta, \\
\cos \theta=\frac{s}{\gamma}, \sin \theta=\frac{\sqrt{\gamma^{2}-s^{2}}}{\gamma}
\end{gathered}
$$

where $\gamma=m_{11}+m_{22}$.

Using Equation (3) for ( $s, \theta)$ dynamics:

$$
d s=\left(-b \gamma \sin \theta-\frac{b}{2} \cos \theta\right) d t+(-\gamma \sin \theta) d w
$$


and for s dynamics using the oil price relation:

$$
d s=\left(-b \sqrt{\gamma^{2}-s^{2}}-\frac{s}{2}\right) d t-\sqrt{\gamma^{2}-s^{2}} d w
$$

\subsubsection{The Lie Group $\mathrm{SO}(3)$}

The Lie group $S O(3)$ is a differential manifold, and it can be identified with unit sphere $S^{2}$. In this manifold, the bilinear state equation, the oil price, and the dynamics for $\mathrm{f}$ are given respectively as follows:

$$
\begin{gathered}
d A=A B d t+A d W, \\
s(A)=\frac{1}{2} \operatorname{Tr}\left(Q A N A^{T}\right), \\
d f=\operatorname{Tr}\left[A^{T} Q A\left((B-I) N d t+d W N+\frac{1}{2} d W N d W^{T}\right)\right]
\end{gathered}
$$

where $A \in S O(3), B, d W \in s o(3)$, and $Q, N \in R^{3 \times 3}$ are positive symmetric matrices.

As a result, the terms in Equation (4) can be defined as follows:

$$
\begin{gathered}
B=\left[\begin{array}{ccc}
0 & -b_{3} & b_{2} \\
b_{3} & 0 & -b_{1} \\
-b_{2} & b_{1} & 0
\end{array}\right] \text { and } d W=\left[\begin{array}{ccc}
0 & -d w_{3} & d w_{2} \\
d w_{3} & 0 & -d w_{1} \\
-d w_{2} & d w_{1} & 0
\end{array}\right] \in \operatorname{so}(3) \\
Q=\left[\begin{array}{lll}
q_{11} & q_{12} & q_{13} \\
q_{12} & q_{22} & q_{23} \\
q_{13} & q_{23} & q_{33}
\end{array}\right], N=\left[\begin{array}{ccc}
n_{11} & n_{12} & n_{13} \\
n_{12} & n_{22} & n_{23} \\
n_{13} & n_{23} & n_{33}
\end{array}\right] \\
\exp \left[\begin{array}{ccc}
0 & -b_{3} & b_{2} \\
b_{3} & 0 & -b_{1} \\
-b_{2} & b_{1} & 0
\end{array}\right]=I_{3}+\sin \theta B\left(b_{1}, b_{2}, b_{3}\right)+(1-\cos \theta) B\left(b_{1}, b_{2}, b_{3}\right)^{2}=A \in S O(3)
\end{gathered}
$$

Hence, we obtain the $s$ oil price and the stochastic dynamic $d s$ for $f$ on $S O(3)$ as follows:

$$
s(A)=\frac{1}{2}\left[c_{11} n_{11}+c_{22} n_{22}+c_{33} n_{33}+\left(c_{12}+c_{21}\right) n_{12}+\left(c_{13}+c_{31}\right) n_{13}+\left(c_{23}+c_{32}\right) n_{23}\right]
$$

where

$$
\begin{gathered}
c_{11}=b_{11} a_{11}+b_{12} a_{21}+b_{13} a_{31}, c_{22}=b_{22} a_{11}+b_{22} a_{22}+b_{23} a_{32} \\
c_{33}=b_{31} a_{13}+b_{32} a_{23}+b_{33} a_{33}, c_{12}=b_{11} a_{12}+b_{12} a_{22}+b_{13} a_{32} \\
c_{21}=b_{21} a_{11}+b_{22} a_{21}+b_{23} a_{31}, c_{13}=b_{11} a_{13}+b_{12} a_{23}+b_{13} a_{33} \\
c_{31}=b_{31} a_{11}+b_{32} a_{21}+b_{33} a_{31}, c_{23}=b_{21} a_{13}+b_{22} a_{23}+b_{23} a_{33} \\
c_{32}=b_{31} a_{12}+b_{32} a_{22}+b_{33} a_{32}
\end{gathered}
$$

and

$$
\begin{aligned}
& b_{11}=a_{11} q_{11}+a_{21} q_{21}+a_{31} q_{31}, b_{12}=a_{11} q_{12}+a_{21} q+a_{31} q_{32} \\
& b_{21}=a_{12} q_{11}+a_{22} q_{21}+a_{32} q_{31}, b_{22}=a_{12} q_{12}+a_{22} q_{22}+a_{23} q_{32} \\
& b_{31}=a_{13} q_{11}+a_{23} q_{21}+a_{33} q_{31}, b_{32}=a_{13} q_{12}+a_{23} q_{22}+a_{33} q_{32} \\
& b_{13}=a_{11} q_{13}+a_{21} q_{23}+a_{31} q_{33}, b_{23}=a_{12} q_{13}+a_{22} q_{23}+a_{32} q_{33} \\
& b_{33}=a_{13} q_{13}+a_{23} q_{23}+a_{33} q_{33} \text { where } a_{i j} \in S O(3) \\
& d s=\operatorname{Tr}\{[\underbrace{A^{T} Q A}_{c_{i j}} \underbrace{B-I) N}_{d_{i j}}] d t+\underbrace{A^{T} Q A}_{c_{i j}} \underbrace{[d W N]}_{e_{i j}}+\underbrace{A^{T} Q A}_{c_{i j}} \underbrace{\left(d W N d W^{T}\right)}_{k_{i j}}\} \\
& =\left[c_{11} d_{11}+c_{12} d_{21}+c_{13} d_{31}+c_{12} d_{12}+c_{22} d_{22}+c_{23} d_{32}+c_{13} d_{13}+c_{23} d_{23}+c_{33} d_{33}\right] d t \\
& +\left[c_{11} e_{11}+c_{12} e_{21}+c_{13} e_{31}+c_{21} e_{12}+c_{22} e_{22}+c_{23} e_{32}+c_{13} e_{13}+c_{23} e_{23}+c_{33} e_{33}\right]
\end{aligned}
$$




$$
\begin{gathered}
+\frac{1}{2}\left[c_{11} k_{11}+c_{12} k_{21}+c_{13} k_{31}+c_{21} k_{12}+c_{22} k_{22}+c_{23} k_{32}+c_{31} k_{13}+c_{32} k_{23}+c_{33} k_{33}\right] \\
d_{i j}=\left(\begin{array}{ccc}
-1 & -b_{3} & b_{2} \\
b_{3} & -1 & -b_{1} \\
-b_{2} & b_{1} & -1
\end{array}\right)\left(\begin{array}{lll}
n_{11} & n_{12} & n_{13} \\
n_{21} & n_{22} & n_{23} \\
n_{31} & n_{32} & n_{33}
\end{array}\right) \\
e_{i j}=\left(\begin{array}{ccc}
0 & -d w_{3} & d w_{2} \\
d w_{3} & 0 & -d w_{1} \\
d w_{2} & d w_{1} & 0
\end{array}\right)\left(\begin{array}{ccc}
n_{11} & n_{12} & n_{13} \\
n_{21} & n_{22} & n_{23} \\
n_{31} & n_{32} & n_{33}
\end{array}\right) \\
k_{i j}=\left(\begin{array}{ccc}
e_{11} & e_{12} & e_{13} \\
e_{21} & e_{22} & e_{32} \\
e_{31} & e_{32} & e_{33}
\end{array}\right)\left(\begin{array}{ccc}
0 & d w_{3} & -d w_{2} \\
-d w_{3} & 0 & d w_{1} \\
d w_{2} & -d w_{1} & 0
\end{array}\right) \\
d s=\left\{\beta(t) s+\alpha_{2} s^{2}\right\} d t+\alpha_{3} s^{3 / 2} d w
\end{gathered}
$$

Thereby, the ds state dynamic obtained by the matrix representation of the Lie group $\mathrm{SO}(3)$ coincides with the state dynamic obtained by the differential representations.

Overall forecasting is formed as

$$
d s \circ L(t)
$$

where $L(t)$ shows long short-term memory (LSTM). LSTM [18] is a recurrent neural network that exploits the dependencies among the samples of a segment of the time series on the $\mathrm{SO}(3)$ manifold for accurate prediction. The equations governing the LSTM operation may be stated as

$$
\begin{gathered}
m_{t}=f_{t} \bigodot_{*} m_{t-1}+j_{t} \bigodot_{*} \widetilde{m}_{t} \\
\widetilde{m}_{t}=\tanh \left(V_{m} x_{t}+W_{m} r_{t-1}+b_{m}\right) \\
j_{t}=\sigma\left(V_{j} x_{t}+W_{j} r_{t-1}+b_{j}\right) \\
f_{t}=\sigma\left(V_{f} x_{t}+W_{f} r_{t-1}+b_{f}\right) \\
o_{t}=\sigma\left(V_{0} x_{t}+W_{o} r_{t-1}+b_{o}\right) \\
r_{t}=o_{t} \bigodot_{*} \tanh \left(m_{t}\right)
\end{gathered}
$$

where $b$ is a bias vector, $W$ and $V$ are weight matrices, the sigmoid function is denoted as $\sigma(\cdot)$, and $\bigodot_{*}$ denotes element-wise multiplication.

The LSTM unit inputs the state vector $m_{t-1}$ and the output vector $r_{t-1}$ from time step $t-1$ as well as the input feature vector $x_{t}$ at time step $t$ to yield the state vector $m_{t}$ and the output vector $r_{t}$ at time step t. Based on $x_{t}$ and $r_{t-1}$, LSTM exploits temporal dependencies by determining the part of the previous state that needs to be kept by using the forget gate $f_{t}$, forming the new information in normalized form as $\widetilde{m}_{t}$, and determining its strength by applying the input gate activation $j_{t}$ to it. The new state is thus formed as $m_{t}$ in Equation (7), which is normalized by tanh function and modulated by the output gate activation $o_{t}$ (Equation (12)) to yield the bounded output prediction as $r_{t}$.

\section{Data and Some Descriptive Statistics}

In this paper, daily West Texas Intermediate (WTI) Crude Oil Prices dataset acquired from the FRED Economic Data. It includes oil price data between 2 January 1986 and 5 April 2021.

The published oil price is the spot price given as

$$
A(t, t+\tau)=E\left[\frac{1}{\tau}\left(\int_{t}^{t+\tau} s(r) d r\right)\right]
$$


As with [15], we have used Monte Carlo simulation to evaluate the above expectation.

Firstly, the descriptive statistics of WTI oil price data were obtained, and unit root test was applied. In Table 1, the statistics are shown. Since the data exhibits excess kurtosis, it cannot be modelled by a normal distribution, as confirmed by the Jarque-Bera (JB) test. The main problem seems to be excess kurtosis but not so much excess skewness.

Table 1. Descriptive statistics and unit root tests.

\begin{tabular}{cc}
\hline Descriptive Statistic & \\
\hline Kurtosis & 28.79 \\
Skewness & 0.983 \\
JB & 1180.97 \\
Observations & 8884 \\
\hline Unit Root Test & \\
\hline ADF & -18.44 \\
KSS & -9.65 \\
\hline
\end{tabular}

From the unit root test results in Table 1, it can be seen that $\mathrm{H}_{0}$ hypothesis can be accepted for all variables at the level. ADF and KSS tests suggest the stationarity of the data at the level.

Next, the results of the nonlinearity tests are presented in Tables 2 and 3. In Table 2, Teraesvirta's neural network test, White neural network test, Likelihood ratio test for threshold nonlinearity, and Tsay's test for nonlinearity indicate that the linear form is mis-specified. Teraesvirta and White tests perform similarly to the Tsay test.

Table 2. Nonlinearity Test Statistics.

\begin{tabular}{cc}
\hline Tests & X-Squared \\
\hline Teraesvirta's neural network test & 26.03616 \\
\hline White neural network test & 20.77706 \\
\hline $\begin{array}{c}\text { Likelihood ratio test for } \\
\text { threshold nonlinearity }\end{array}$ & 360.5499 \\
\hline Tsay's test for nonlinearity & F-statistics \\
\hline
\end{tabular}

The R software nonlinearTseries package [19] for calculating Nonlinearity Test Statistics.

Table 3. BDS test statistic.

\begin{tabular}{cc}
\hline Dimension & $z$-Statistic \\
\hline $\mathbf{2}$ & 276.5526 \\
\hline $\mathbf{3}$ & 297.6034 \\
\hline $\mathbf{4}$ & 323.5149 \\
\hline $\mathbf{5}$ & 360.5517 \\
\hline $\mathbf{6}$ & 410.8878 \\
\hline
\end{tabular}

The BDS test (Brock et al. [20]) in Table 3 suggests that the (linear) functional form is misspecified for the variables.

\section{Models and Results}

The Lie parameters in Equation (5) were obtained by using the OLS and NLS methods. Table 4 shows estimates of the Lie parameters. The $\alpha_{2}$ coefficient estimates obtained with the two methods turned out to be very similar, whereas $\alpha_{3}$ coefficient estimates obtained 
with the two methods were significantly different. The AIC values obtained with both models are similar to each other.

Table 4. Estimations of Lie parameters.

\begin{tabular}{|c|c|c|}
\hline & \multicolumn{2}{|c|}{ Lie Methods } \\
\hline & Lie-OLS & Lie-NLS \\
\hline$\alpha_{2}$ & $\begin{array}{l}-0.319 \\
(-5.18)\end{array}$ & $\begin{array}{c}-0.321 \\
(2.58)\end{array}$ \\
\hline$\alpha_{3}$ & $\begin{array}{c}-1.067 \\
(3.16)\end{array}$ & $\begin{array}{c}-0.321 \\
(2.58)\end{array}$ \\
\hline$\beta_{1}$ & $\begin{array}{l}0.256 \\
(1.89)\end{array}$ & $\begin{array}{l}0.526 \\
(4.80)\end{array}$ \\
\hline$\beta_{2}$ & $\begin{array}{c}-0.3048 \\
(1.73)\end{array}$ & $\begin{array}{c}-0.3048 \\
(-2.56)\end{array}$ \\
\hline$\beta_{3}$ & $\begin{array}{l}0.474 \\
(2.36)\end{array}$ & $\begin{array}{l}-0.282 \\
(14.96)\end{array}$ \\
\hline$\beta_{4}$ & $\begin{array}{l}-0.616 \\
(3.209)\end{array}$ & $\begin{array}{l}0.4616 \\
(3.21)\end{array}$ \\
\hline$\beta_{5}$ & $\begin{array}{c}-0.282 \\
(2.64)\end{array}$ & $\begin{array}{l}0.4746 \\
(1.97)\end{array}$ \\
\hline AIC & -11.89 & -11.65 \\
\hline $\mathbf{R}^{2}$ & 0.66 & 0.78 \\
\hline Log likelihood & 52.828 & 79.651 \\
\hline ARCH & $3.79 *$ & 2.45 \\
\hline BP & $9.48 *$ & 2.99 \\
\hline Keenan & 3.16 & 1.54 \\
\hline RESET & $3.81 *$ & 2.8 \\
\hline
\end{tabular}

*ARCH test is Engle's test for first order ARCH. BP is the Breusch-Pagan test for heteroscedasticity. Keenan test and RESET test are tests for non-linearity.

It is interesting that Lie-OLS model passes RESET, BP, and ARCH tests with values very close to the critical value. On the other hand, the statistical tests of the Lie $\mathrm{NLS}_{\mathrm{S}}$ model gave more successful results than the statistical tests of the Lie-OLS model.

Next, the forecasting performances with the Lie $\mathrm{OLS}_{\mathrm{S}}$ and Lie $\mathrm{NLS}_{\mathrm{S}}$ models were analyzed. LSTM was used to improve the forecasting performances of these models. In order to apply the LSTM model, the dataset was partitioned into an in-sample training set and out-sample test set corresponding to the time intervals between 2 January 1986-20 October 2019 and 21 October 2019-5 April 2021, respectively.

The configuration of our LSTM network is as follows:

- Input samples consist of sequence segments of 30 timesteps, each having 1 feature (price).

- Input layer is connected to an LSTM unit with 25 hidden neurons and a dropout value of 0.20 .

- LSTM output feeds a dense layer (output) with one neuron and linear activation function

- Training is performed in batches of 32 samples.

The model giving the lowest RMSE and MAE values is deemed the most successful model.

\subsection{In-Sample Forecast Results}

Table 5 presents the results of the LSTM method integrated with the Lie ${ }_{\mathrm{OLS}}$ model or 
the $\mathrm{Lie}_{\mathrm{NLS}}$ model. As references for comparison, the results with the Lie $\mathrm{OLS}$ and Lie $\mathrm{NLS}$ models (employing traditional regression techniques) are also presented.

Table 5. In-sample forecast results.

\begin{tabular}{ccccc}
\hline & Traditional Lie Method & \multicolumn{2}{c}{ Lie Deep Method } & \\
\hline & Lie $_{\text {OLS }}$ & Lie $_{\text {NLS }}$ & Lie-LSTM $_{\text {OLS }}$ & Lie-LSTM $_{\text {NLS }}$ \\
\hline MAE & 0.08300 & 0.1640 & 0.007676 & 0.002671 \\
\hline RMSE & 0.10223 & 0.2027 & 0.011423 & 0.006425 \\
\hline
\end{tabular}

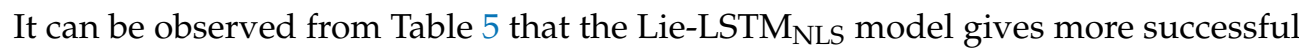
results than the Lie-LSTM OLS $_{\text {model. More importantly, it is also seen that Lie-LSTM }}$ OLS and Lie-LSTM $M_{N L S}$ models give more successful results than the Lie OLS $_{\text {and Lie }}$ NLS models used for reference.

\subsection{Out-of-Sample Forecast Results}

The RMSE and MAE values for Lie $_{\mathrm{OLS}}, \mathrm{Lie}_{\mathrm{NLS}}, \mathrm{Lie}_{\mathrm{LSTM}}$ OLS, and Lie-LSTM $\mathrm{NLS}$ were obtained to explore their forecast accuracy for T+10 and T+20 workdays in Table 6 .

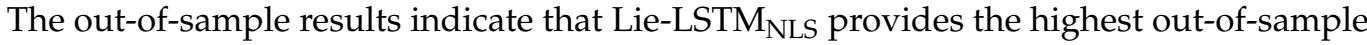
forecast accuracy.

Table 6. The out-of-sample performances of compared methods.

\begin{tabular}{ccccccc}
\hline \multicolumn{7}{c}{ Traditional Lie Methods } \\
\hline & Lie OLS & & Lie $_{\text {NLS }}$ & \\
\hline & $\mathbf{T + 1}$ & $\mathbf{T + 1 0}$ & $\mathbf{T + 2 0}$ & $\mathbf{T + 1}$ & $\mathbf{T + 1 0}$ & $\mathbf{T + 2 0}$ \\
\hline MAE & 0.0495 & 0.051 & 0.058 & 0.076 & 0.078 & 0.083 \\
\hline RMSE & 0.0633 & 0.066 & 0.069 & 0.088 & 0.092 & 0.095 \\
\hline \multicolumn{7}{c}{ Deep Neural Networks } \\
\hline MAE & 0.014085 & 0.014168 & 0.014636 & 0.002710 & 0.002174 & 0.001601 \\
\hline RMSE & 0.028279 & 0.028653 & 0.029099 & 0.007710 & 0.008449 & 0.008548 \\
\hline
\end{tabular}

\subsection{Test for Forecast Accuracy}

The Wilcoxon signed-rank and Diebold-Mariano (DM) tests were applied to test the equivalence of forecast accuracy (null hypothesis $\mathrm{H}_{0}$ ).

In Tables 7 and 8, the $p$ values of calculated DM and Wilcoxon test statistics are 0.00 , and both of them are significant at the $1 \%$ significance level. $\mathrm{The}_{0} \mathrm{H}_{0}$ hypothesis of these tests assume the models have the same level of accuracy. For most cases, since the $p$-value is $<0.05, \mathrm{H}_{0}$ hypothesis is rejected. For both tests, the $p$-value is $>0.05$ only for the RMSE comparison of the Lie $\mathrm{OLS}$ and Lie $\mathrm{NLS}_{\mathrm{S}}$ models. Hence, these two models are comparable in terms of RMSE performance.

Table 7. $p$-values for the Wilcoxon signed-rank tests.

\begin{tabular}{ccccc}
\hline & Lie $_{\text {OLS }}$ & Lie $_{\text {NLS }}$ & Lie-LSTM $_{\text {OLS }}$ & Lie-LSTM $_{\text {NLS }}$ \\
\hline Lie $_{\text {OLS }}$ & - & & & \\
\hline Lie $_{\text {NLS }}$ & 0.41 & - & & \\
\hline Lie-LSTM $_{\text {OLS }}$ & 0.00 & 0.00 & 0.00 & - \\
\hline Lie-LSTM $_{\text {NLS }}$ & 0.00 & 0.00 & & \\
\hline
\end{tabular}


Table 8. $p$-values for the Diebold-Mariano tests.

\begin{tabular}{ccccc}
\hline & Lie $_{\text {OLS }}$ & Lie $_{\text {NLS }}$ & Lie-LSTM $_{\text {OLS }}$ & Lie-LSTM $_{\text {NLS }}$ \\
\hline Lie $_{\text {OLS }}$ & - & & & \\
\hline Lie $_{\text {NLS }}$ & 0.32 & - & & \\
\hline Lie-LSTM $_{\text {OLS }}$ & 0.00 & 0.00 & - & - \\
\hline Lie-LSTM $_{\text {NLS }}$ & 0.00 & 0.00 & 0.00 & \\
\hline
\end{tabular}

\section{Discussion and Conclusions}

We have proposed hybrid models for analyzing the short-term model of the oil during the period from 2 January 1986 to 5 April 2021. In our basic model, the Lie group SO(3) is a differential manifold, and it can be identified with unit sphere $S^{2}$. We have additionally integrated this model with LSTM to test it.

The previous works by [8,9] differ significantly from the current work since [8] only discussed the mean reverting tendency of oil price, and the model by [9] depends on a twofactor model for pricing financial and real assets contingent on the price of oil. Specifically, it estimates the parameters of joint stochastic processes modelling oil-contingent claim and futures contract based on spot prices and net convenience yield and uses this model to value futures contracts. On the other hand, our work develops the short-term model and solves it with the hybrid model of Lie method and LSTM network. Although Lie algebras have been used in interest rate models in many works in the literature, the current work is the first to use Lie algebras for oil price modelling. When the time series for oil prices are considered, it is seen that their distributions have positive skewness. Even though some other methods might be tried out to perform analyses on these processes, this problem can be readily addressed with our proposed Lie model. This can be attributed to the modelling of the oil price on the $S^{2}$ manifold using matrix representations and differential operators in our suggested Lie $\mathrm{OLS}_{\mathrm{S}}$ and $\mathrm{Lie}_{\mathrm{NLS}}$ models. Then, we maintain that carrying out a modelling with the Lie-LSTM methods obtains good forecasting results.

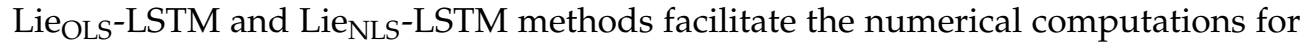
stochastic differential equations on differentiable manifolds. By employing $\mathrm{SO}(3)$ group structure, the oil prices that have a positive skewness and high JB and kurtosis can be described in a geometric way. Moreover, by jointly using the Lie and LSTM methods, it becomes possible to increase forecasting performance by representing the complex structure.

As stated in Park et al. [15] for interest rate, the Lie group models in the current work show that the closed form formulas can only be an exception rather than the rule for oil price prediction, and therefore one should resort to numerical approaches for such prediction. Additionally, while the previous works ([15,16] for bond pricing) have employed either OLS or NLS methods to estimate the model parameters, the current work investigated which of these methods works best with the Lie method. Each of these combinations, Lie $\mathrm{OLS}_{\text {and }} \mathrm{Lie}_{\mathrm{NLS}}$, were integrated with the LSTM network to get a hybrid model with improved forecasting performance. Specifically, in price forecasting 10 and 20 days into the future, the models incorporating LSTM yielded smaller RMSE and MAE values. According to Wilcoxon signed-rank and Diebold-Mariano tests, Lie-LSTM NLS $_{2}$ model turned out to be the most successful one in terms of forecasting performance among the four models considered.

In this study, we showed that the analysis of the short-term model of the spot price of oil by using the Lie method is important. The model that we propose can also be used to analyze the relationship between futures and spot prices of many commodities other than oil.

Author Contributions: Conceptualization, M.B., N.G.B. and Y.U.; methodology, M.B., N.G.B. and Y.U.; software, M.B., N.G.B. and Y.U.; validation, M.B., N.G.B. and Y.U.; formal analysis, M.B., N.G.B. and Y.U.; investigation, M.B., N.G.B. and Y.U.; resources, M.B., N.G.B. and Y.U.; data curation, M.B., N.G.B. and Y.U.; writing-original draft preparation, M.B., N.G.B. and Y.U.; writing—review and 
editing, M.B., N.G.B. and Y.U.; visualization, M.B., N.G.B. and Y.U.; supervision, M.B., N.G.B. and Y.U.; project administration, M.B., N.G.B. and Y.U.; funding acquisition, M.B., N.G.B. and Y.U. All authors have read and agreed to the published version of the manuscript.

Funding: This research received no external funding.

Data Availability Statement: The WTI dataset can be downloaded from https:/ / fred.stlouisfed.org/ series/DCOILWTICO, accessed on 15 April 2021.

Conflicts of Interest: The authors declare no conflict of interest.

\section{References}

1. Barone-Adesi, G.; Bourgoin, F.; Giannopoulos, K. Don't look back. Risk 1998, 11, 100-103.

2. Adrangi, B.; Chatrath, A.; Dhanda, K.K.; Raffiee, K. Chaos in oil prices? Evidence from futures markets. Energy Econ. 2001, 23, 405-425. [CrossRef]

3. Lahmiri, S. A study on chaos in crude oil markets before and after 2008 international financial crisis. Phys. A Stat. Mech. Its Appl. 2017, 466, 389-395. [CrossRef]

4. Bildirici, M.E.; Sonustun, F.O. Chaotic Structure of Oil Prices, Inflation and Unemployment. Nonlinear Dyn. Psychol. Life Sci. 2019, 23, 377-394.

5. Komijani, A.; Naderi, E.; Gandali Alikhani, N. A hybrid approach for forecasting of oil prices volatility. OPEC Energy Rev. 2014, 38, 323-340. [CrossRef]

6. He, L.-Y. Chaotic Structures in Brent \& WTI Crude Oil Markets: Empirical Evidence. Int. J. Econ. Financ. 2011, 3, 242-249. [CrossRef]

7. Bildirici, M.; Guler Bayazit, N.; Ucan, Y. Analyzing Crude Oil Prices under the Impact of COVID-19 by Using LSTARGARCHLSTM. Energies 2020, 13, 2980. [CrossRef]

8. Gibson, R.; Schwartz, E. Valuation of Long Term Oil-Linked Assets; Working Paper; Anderson Graduate School of Management, University of California: Los Angeles, CA, USA, 1989; Volume 6, p. 89.

9. Gibson, R.; Schwartz, E.S. Stochastic convenience yield and the pricing of oil contingent claims. J. Financ. 1990, 45, 959-976. [CrossRef]

10. Nunes, J.; Webber, N.J. Low Dimensional Dynamics and the Stability of HJM Term Structure Models; Working Paper; AIP Publishing: Melville, NY, USA, 1997.

11. Gazizov, R.K.; Ibragimov, N.H. Lie symmetry analysis of differential equations in finance. Nonlinear Dyn. 1998, 17, 387-407. [CrossRef]

12. Ibragimov, N.H.; Soh, C.W. Solution of the Cauchy problem for the Black-Scholes equation using its symmetries. In Proceedings of the Modern Group Analysis, International Conference at the Sophus Lie Conference Center, Nordfjordeid, Norway, 9-13 June 1997.

13. Lo, C.F.; Hui, C.H. Valuation of financial derivatives with time-dependent parameters: $\{$ Lie\}-algebraic approach. Quant. Financ. 2001, 1, 73-78. [CrossRef]

14. Carr, P.; Lipton, A.; Madan, D. The Reduction Method for Valuing Derivative Securities; Working Paper; New York University: New York, NY, USA, 2002.

15. Park, F.C.; Chun, C.M.; Han, C.W.; Webber, N. Interest rate models on Lie groups. Quant. Financ. 2011, 11, 559-572. [CrossRef]

16. Goard, J. New solutions to the bond-pricing equation via Lie's classical method. Math. Comput. Model. 2000, 32, 299-313. [CrossRef]

17. Klimyk, A.U.; Vilenkin, N.Y. Representations of Lie groups and special functions. In Representation Theory and Noncommutative Harmonic Analysis II; Springer: Berlin/Heidelberg, Germany, 1995; pp. 137-259.

18. Hochreiter, S.; Schmidhuber, J. Long short-term memory. Neural Comput. 1997, 9, 1735-1780. [CrossRef] [PubMed]

19. Garcia, C.A. NonlinearTseries: Nonlinear Time Series Analysis; R Foundation for Statistical Computing: Vienna, Austria, 2020.

20. Brock, W.; Dechert, W.D.; Scheinkman, J. A Test for Independence Based on the Correlation Dimension; Economy Working Paper SSRI-8702; University of Wisconsin: Madison, WI, USA, 1987. 